\title{
LETTER
}

\section{A human model of intra-abdominal hypertension: even slightly elevated pressures lead to increased acute systemic inflammation and signs of acute kidney injury}

\author{
Marije Smit*1, H Sijbrand Hofker ${ }^{2}$, Henri GD Leuvenink², Christina Krikke², Rianne M Jongman³ , Jan G Zijlstra' \\ and Matijs van Meurs ${ }^{1}$
}

Intra-abdominal hypertension (IAH) may have catastrophic effects on critically ill patients, but its pathophysiology is only partially understood [1]. Oliguria and renal dysfunction are among the earliest signs of increasing intra-abdominal pressure (IAP) [2]. A number of different pathophysiological mechanisms may be responsible [1]. One of these may be an increase in pro-inflammatory cytokines provoked by IAH [2]. In IAPs of greater than $20 \mathrm{~mm} \mathrm{Hg}$, an increase in circulating levels of a variety of inflammatory mediators has been described [3].

We analyzed markers of inflammation and renal function in a prospective randomized clinical trial. In this trial, 50 living kidney donors were randomly assigned between hand-assisted laparoscopy by a transperitoneal approach and open nephrectomy by a retroperitoneal approach [4]. In this human model of IAH, a carbon dioxide pneumo-peritoneum of $12 \mathrm{~mm} \mathrm{Hg}$ was induced during the procedure in the laparoscopic group. The open nephrectomy group served as a control group, and IAH was not applied.

Markers of systemic inflammation - C-reactive protein (CRP) and interleukin-6 (IL-6) - were determined in both groups peri-operatively. These results were previously published in a large study on the effect of different operative procedures on outcome of kidney donors [4]. As a marker of renal function, neutrophil gelatinaseassociated lipocalin (NGAL) in plasma and urine was determined in both groups at the start of the procedure and just before extraction of the donor kidney. All data are expressed as means with standard deviations. Paired

\footnotetext{
*Correspondence: m.smit@umcg.nl

'Department of Critical Care, University Medical Center Groningen, University of Groningen, Hanzeplein 1, 9713 GZ Groningen, The Netherlands

Full list of author information is available at the end of the article
}

$t$ tests were performed to test for statistical significance at every time point; a $P$ value of less than 0.05 was considered statistically significant. CRP was 1.5 times higher after 24 hours and 1.3 times higher after 48 hours in the laparoscopic group. IL-6 was 1.5 times higher after 12 hours in the laparoscopic group. NGAL in plasma was 1.2 times higher just before extraction of the kidney in the laparoscopic group. NGAL in urine did not change (Figure 1).

Although procedure time is shorter [4], laparoscopic nephrectomy leads to increased inflammation and signs of renal injury. It is tempting to hypothesize that even mild IAH leads to mildly increased systemic inflammation and increased plasma NGAL as a sign of acute kidney injury in this group. Other factors such as operative technique or inflammation caused by carbon dioxide gas may contribute. Further studies to dissect the contributions of inflammation, hemodynamics, and local pressure effects on renal injury in IAH are warranted.

\section{Abbreviations}

CRP, C-reactive protein; IAH, intra-abdominal hypertension; IAP, intra-

abdominal pressure; IL-6, interleukin-6; NGAL, neutrophil gelatinase-associated lipocalin.

\section{Competing interests}

The authors declare that they have no competing interests.

\section{Acknowledgments}

The study was approved by the Medical Ethical Review Board of the University Medical Center Groningen and conducted in accordance with the principles of the 2000 Declaration of Helsinki. The trial was registered at ClinicalTrials.gov (NCT00258986). Informed consent for participation and for publication was obtained from all patients before inclusion.

\section{Author details}

'Department of Critical Care, University Medical Center Groningen, University of Groningen, Hanzeplein 1, 9713 GZ Groningen, The Netherlands. 2Department of Surgery, University Medical Center Groningen, University of Groningen, Hanzeplein 1, 9713 GZ Groningen, The Netherlands. ${ }^{3}$ Departments of Anesthesiology and Pathology and Medical Biology, Medical Biology Section, University Medical Center Groningen, University of Groningen, Hanzeplein 1,9713 GZ Groningen, The Netherlands. 


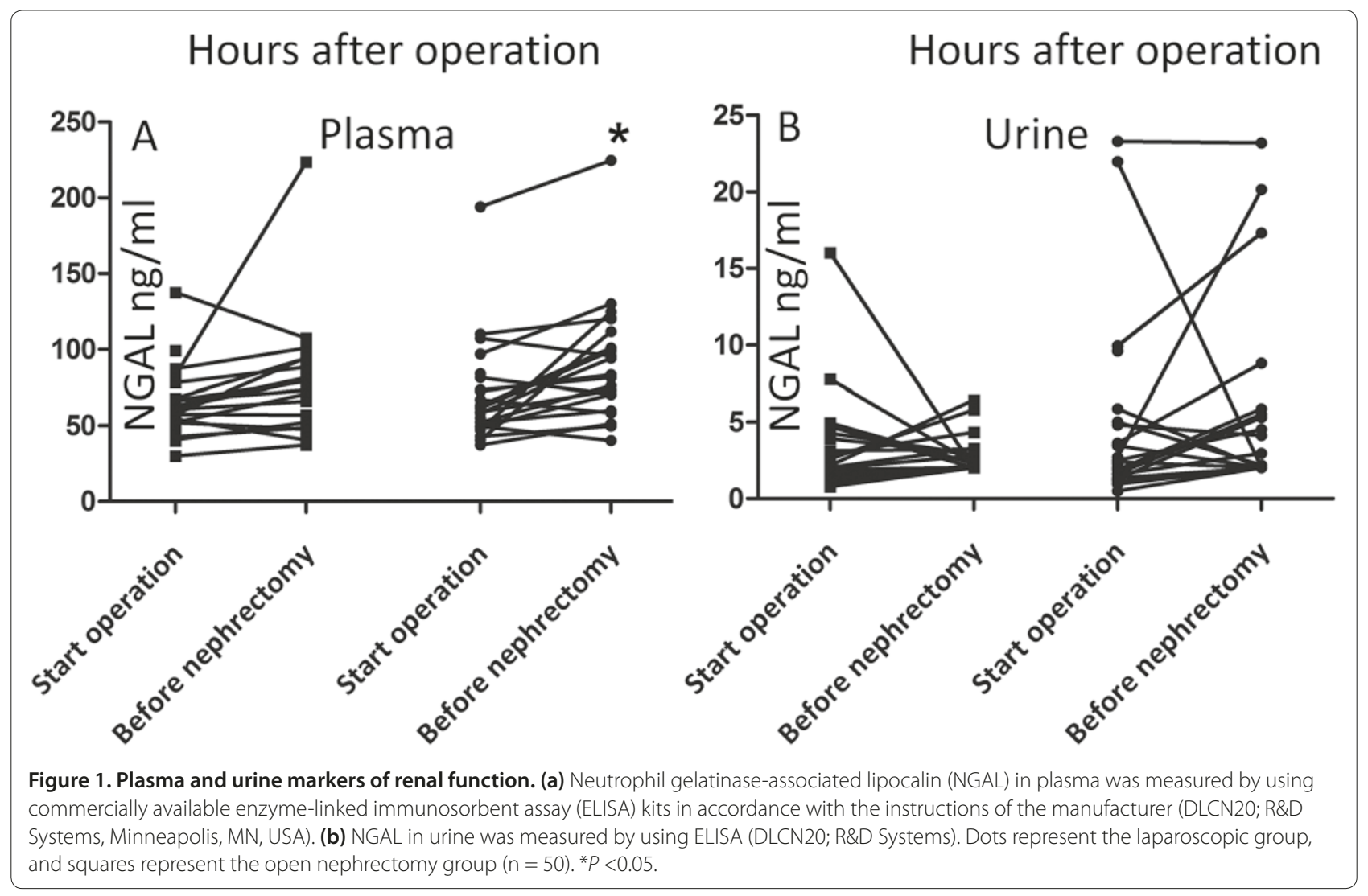

Published: 4 April 2013

\section{References}

1. Malbrain ML, Vidts W, Ravyts M, De Laet I, De Waele J: Acute intestinal distress syndrome: the importance of intra-abdominal pressure. Minerva Anestesiol 2008, 74:657-673.

2. Mohmand H, Goldfarb S: Renal dysfunction associated with intraabdominal hypertension and the abdominal compartment syndrome. J Am Soc Nephrol 2011, 22:615-621.

3. Rezende-Neto JB, Moore EE, Melo de Andrade MV, Teixeira MM, Lisboa FA, Arantes RM, de Souza DG, da Cunha-Melo JR: Systemic inflammatory response secondary to abdominal compartment syndrome: stage for multiple organ failure. J Trauma 2002, 53:1121-1128.
4. Hofker HS, Nijboer WM, Niesing J, Krikke C, Seelen MA, van Son WJ, van Wijhe $\mathrm{M}$, Groen H, vd Heide JJ, Ploeg RJ: A randomized clinical trial of living donor nephrectomy: a plea for a differentiated appraisal of mini-open muscle splitting incision and hand-assisted laparoscopic donor nephrectomy. Transplant Int 2012, 25:976-986.

doi:10.1186/cc12568

Cite this article as: Smit M et al: A human model of intra-abdominal hypertension: even slightly elevated pressures lead to increased acute systemic inflammation and signs of acute kidney injury. Critical Care 2013, 17:425. 